\title{
Simulation of Metal Transfer in GMAW Based on FLUENT
}

Xueping DING ${ }^{1)}$, Huan $L I^{1)}$, Lijun $Y A N G^{1) \dagger}$ and Ying $G A O^{2)}$

1) Tianjin Key Laboratory of Advanced Joining Technology, Tianjin University, Tianjin 300072, China

2) Tianjin Key Laboratory of High Speed Cutting and Precision Machining, Tianjin University of Technology and Education, Tianjin 300222, China

[Manuscript received 16 October 2012, in revised form 9 January 2013]

(C) The Chinese Society for Metals and Springer-Verlag Berlin Heidelberg

\begin{abstract}
A new numerical approach is presented, which is used to simulate the dynamic process of metal transfer. The process of metal transfer in gas metal arc welding is simulated based on FLUENT. A two-dimensional axisymmetric numerical model is developed using volume of fluid method and the distributions of physical quantities including pressure, current density, electric potential in the droplet are investigated. For improving the veracity of the simulated results and decreasing the effect of the uncertain surface tension coefficient on the simulated results, the relationship between the welding current and surface tension coefficient is modified by analysis of regression. Meanwhile for testing the accuracy of simulated results, the welding experiments are performed and the high-speed photography system is used to record the real process of metal transfer. The results show that the simulated results are in reasonably good agreement with the experimental ones.
\end{abstract}

KEY WORDS: Metal transfer; FLUENT; Dynamic simulation; High-speed photography

\section{Introduction}

Gas metal arc welding (GMAW) is widely applied in industry because of its high efficiency and excellent quality. In the welding process, due to different welding parameters including wire composition, diameter, shielding gas composition, electrical polarity, as well as arc length and welding current level, metal transfer can be divided into contact transfer and free-flight transfer ${ }^{[1]}$. As the metal transfer process plays an important role in arc stability and weld quality, it is meaningful to investigate the metal transfer phenomenon ${ }^{[2]}$.

Thanks to the remarkable progress of computer technology, it has promoted a deeper understanding of metal transfer process by means of numerical calculations. Various scholars have done a lot work. Concerning the simulation of contact transfer, Choi et al. ${ }^{[3]}$ proposed a short circuit model to predict the variation of short circuit parameters considering the effects of the surface tension and electromagnetic force and the calculated results are in broad agreement with

\footnotetext{
† Corresponding author. Associate Prof., Ph.D.; Tel: + 8622 27406261; Fax: +86 022 27404724;

E-mai address: yljabc@tju.edu.cn (Lijun YANG)
}

the experimental results that occur with argon shielding but then there were few researchers to make a study of this. In fact, many researchers were apt to simulate the free-flight transfer such as globular and spray transfer. They have envisaged the metal transfer process based on static force balance model ${ }^{[4,5]}$ and dynamic force balance model ${ }^{[6]}$. There were also some researchers who simulated the droplet detachment and oscillation on the basis of spring-mass theory ${ }^{[7-12]}$. Moreover, some numerical models based on volume of fluid (VOF) method were developed to simulate metal transfer combined with fluid flow, heat transfer, arc plasma, anode sheath, metal vapor and so on ${ }^{[13-25]}$. Praveen et al. ${ }^{[26]}$ developed a statistical model and predict the transfer mode in pulse GMAW of aluminum quantitatively. Semenov et al. ${ }^{[27]}$ pointed out the defects of the hydrostatical model and the complete hydrodynamic model and proposed the approximate hydrodynamic model which allowed the prediction of droplet geometry right up to its detachment. But the simulations mentioned above were achieved mostly by means of programming language. It is complex and difficult for the newcomers. The researchers are required to have good programming basis and it will take long cycle to finish the programming. Besides if some errors appear, the 
researchers have to spend long time to debug. In brief, programming is inefficient.

Thus, commercial computational fluid dynamics (CFD) software, FLUENT, is utilized to simulate the metal transfer process in this paper. FLUENT is a powerful modeling tool combining fluid dynamics and computer technology. Adopting this software to replace programming, it will save energy of researchers so they can concentrate on studying the mechanism of the metal transfer, modifying the physical model and improving accuracy of simulated results. In this study, the process of metal transfer in GMAW is simulated. The VOF model of two-phase flow module is utilized to distinguish two immiscible fluids (shielding gas and molten metal) and to track the interface between them. In addition, the relationship between welding current and surface tension coefficient is obtained by analysis of regression.

Meanwhile, to assess validity of the simulated results, welding experiments were performed. The results show that simulated results are in reasonable good agreement with experimental ones and it improves our understanding of metal transfer process in GMAW and assesses modeling capability of FLUENT in welding simulation.

\section{Mathematical Model}

In order to simulate the complex metal transfer process, some assumptions are given below: (1) The molten metal is a incompressible and newtonian fluid; (2) Materials properties of molten steel are assumed to be constant; (3) The thermal analysis and the effect of arc on metal transfer are neglected ${ }^{[18]}$. The above assumptions may affect the metal transfer to some extent. Future studies on these factors are recommended to be made.

The metal transfer process in GMAW is analogous to that of water dripping or flowing from an orifice, except that the driving force for the flow is primarily electromagnetic rather than gravity ${ }^{[28]}$. In this model, the electrode is assumed to be at the molten temperature and the velocity of molten metal is assumed to be equal to the wire feed rate ${ }^{[20]}$. A two-dimensional axisymmetric model in FLUENT is shown in Fig. 1. In the model, $h$ and $w$ is the height and width of the model respectively and the value of them depend on the arc voltage. There are two phases in the computational domain: a liquid phase (shadow area - molten metal) and a gas phase (blank area-shielding gas). In the simulation, as the model is axisymmetric, we just select half of the model to calculate. The length and width of the model is $10 \mathrm{~mm}$ and $8 \mathrm{~mm}$, respectively.

Based on the above mentioned assumptions, the following differential equations governing the conservation of mass, momentum and current continuity equation are given below:

(1) Mass continuity equation

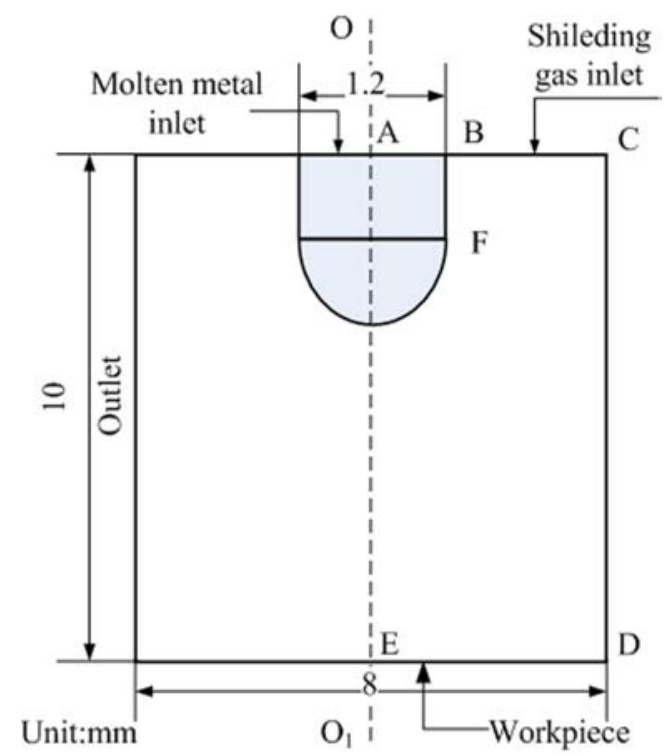

Fig. 1 Schematic for metal transfer modeling (unit: $\mathrm{mm}$ )

$$
\frac{\partial \rho}{\partial t}+\frac{1}{r} \frac{\partial(r \rho u)}{\partial r}+\frac{\partial(\rho v)}{\partial z}=0
$$

(2) Momentum equation

$$
\begin{aligned}
& \frac{\partial(\rho u)}{\partial t}+\frac{1}{r} \frac{\partial}{\partial r}(\rho r u u)+\frac{\partial}{\partial z}(\rho u v)=f_{\mathrm{r}}-\frac{\partial P}{\partial r}+ \\
& \frac{1}{r} \frac{\partial}{\partial r}\left(u r \frac{\partial u}{\partial r}\right)-2 \mu \frac{u}{r^{2}}+\frac{\partial}{\partial z}\left(\mu \frac{\partial v}{\partial r}\right) \\
& \frac{\partial(\rho v)}{\partial t}+\frac{1}{r} \frac{\partial}{\partial r}(\rho r u v)+\frac{\partial}{\partial z}(\rho v v)=f_{\mathrm{z}}-\frac{\partial P}{\partial z}+ \\
& \frac{1}{r} \frac{\partial}{\partial r}\left(\mu r \frac{\partial u}{\partial z}\right)+\frac{\partial}{\partial z}\left(\mu \frac{\partial v}{\partial z}\right)
\end{aligned}
$$

(3) Current continuity equation

$$
\frac{\partial}{\partial z}\left(\sigma \frac{\partial \varphi}{\partial z}\right)+\frac{1}{r} \frac{\partial}{\partial r}\left(\sigma r \frac{\partial \varphi}{\partial r}\right)=0
$$

In the above equations, $u$ and $v$ are the velocities in the respective $r$ and $z$ directions. $\rho, \mu$ and $P$ are the mass density, kinematic viscosity and hydrodynamic pressure, respectively. $\varphi$ is the scalar of electric potential. $f_{\mathrm{r}}$ and $f_{\mathrm{z}}$ are the body force which include the gravity, electromagnetic force, and plasma flow force. The detailed equations for the above body force refer to Ref. [4].

In the simulation, the VOF model in two-phase flow is used to track the free surface of the droplet. $F$ denotes a volume function that is used to track the profile of free surfaces and it satisfies the following conservation equation

$$
\frac{\mathrm{d} F}{\mathrm{~d} t}=\frac{\partial F}{\partial t}+(V \cdot \nabla) F=0
$$

The scalar function, $F$, is defined as the fractional volume of the droplet fluid in the respective cells of the computational mesh. When $F$ is equal to 1 , it 
corresponds to a cell which is full of molten metal. When $F$ is equal to 0 , it indicates the cell contains no molten metal. Cells with $F$ values between 0 and 1 are partially filled with molten metal ${ }^{[29]}$.

The differential equations above mentioned are discretized and solved iteratively by the CFD commercial software FLUENT code, improved with dedicated user defined function (UDF) to solve the source terms and the user defined scalar (UDS) needed for solving the current continuity equation.

In the model, the boundary conditions are shown in Table $1 . U_{\mathrm{c}}$ is a constant voltage, $v_{\mathrm{f}}$ is the wire feed rate and $v_{\mathrm{g}}$ is the shielding gas velocity. An initial velocity of $1 \mathrm{~m} / \mathrm{s}$ and voltage of $10 \mathrm{~V}$ are set to ensure the convergence.

Table 1 Boundary conditions of the model

\begin{tabular}{ccccc}
\hline Boundary & $u$ & $v$ & $\varphi$ & $F$ \\
\hline $\mathrm{A} / \mathrm{B}$ & 0 & $v_{\mathrm{f}}$ & $U_{\mathrm{c}}$ & 1 \\
$\mathrm{~B} / \mathrm{C}$ & 0 & $v_{\mathrm{g}}$ & $\frac{\partial \varphi}{\partial z}=0$ & 0 \\
$\mathrm{C} / \mathrm{D}$ & $\frac{\partial u}{\partial r}=0$ & 0 & $\frac{\partial \varphi}{\partial r}=0$ & 0 \\
$\mathrm{D} / \mathrm{E}$ & 0 & 0 & 0 & 0 \\
\hline
\end{tabular}

Simulations are performed for a mild steel electrode with $1.2 \mathrm{~mm}$ diameter and the shielding gas $\mathrm{Ar}$ is supplied with a flow rate of $15 \mathrm{~L} / \mathrm{min}$. Material properties of the molten steel used for the simulation are listed in Table $2^{[16]}$.

Table 2 Material properties used in simulation

\begin{tabular}{cc}
\hline Property & Value \\
\hline Mass density $\rho$ & $7860 \mathrm{~kg} \cdot \mathrm{m}^{-3}$ \\
Kinematic viscosity $\mu$ & $2.8 \times 10^{-7} \mathrm{~m}^{2} \cdot \mathrm{s}^{-1}$ \\
Magnetic permeability $\mu_{0}$ & $4 \pi \times 10^{-7} \mathrm{H} \cdot \mathrm{m}^{-1}$ \\
Gravitational acceleration $g$ & $9.8 \mathrm{~m} \cdot \mathrm{s}^{-2}$ \\
Electrical conductivity $\sigma$ & $7.7 \times 10^{5} \Omega^{-1} \cdot \mathrm{m}^{-1}$ \\
\hline
\end{tabular}

\section{Results and Discussion}

The profiles of the metal transfer process are shown in Fig. 2 and Fig. 3. Observing the metal transfer process in Fig. 2, at first, the droplet grows slowly, and then as time goes on, a lot liquid metal accumulates at the electrode tip. Then due to the large mass of the liquid metal, the neck forms. When the liquid droplet is about to break away from the electrode at $t=148.6 \mathrm{~ms}$, the part of the droplet below the liquid bridge approximates a spherical shape and the remaining liquid connected to the electrode presents a conical shape. With the action of gravity, the surface tension and the electromagnetic force, the droplet detaches from the electrode tip. Meanwhile, once the liquid bridge that connects the falling droplet to the remaining liquid at the electrode tip breaks, except the large volume droplet, the breaking of liquid bridge will produce one or more small droplets

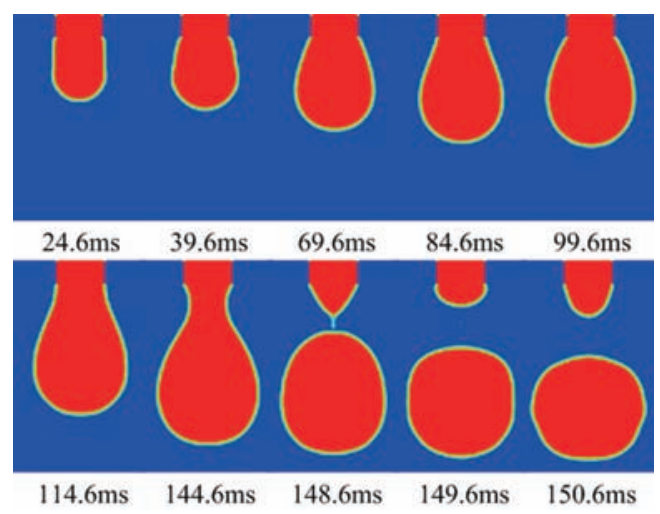

Fig. 2 Simulated results for the metal transfer $(I=175 \mathrm{~A})$

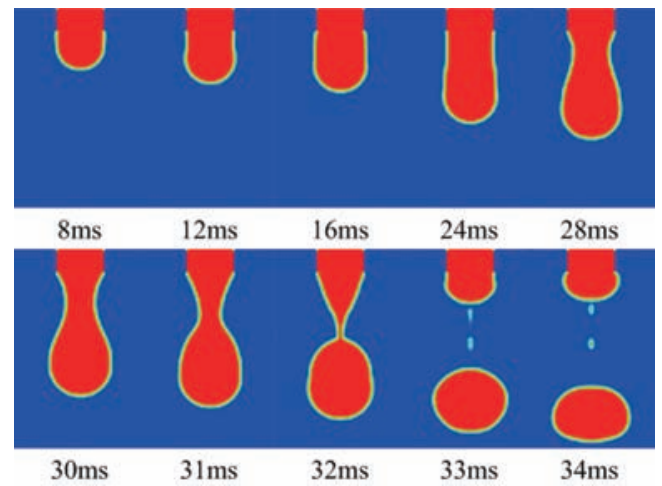

Fig. 3 Simulated results for the metal transfer $(I=210 \mathrm{~A})$

which are named satellite droplets in Ref. [18]. In fact, these satellite droplets are the reason for the production of the spatter in the welding process. After the droplet detaches, due to the severe deformation of the surface, a large surface tension on the liquid that remains at the electrode tip is generated. The surface tension leads to the liquid to recoil and oscillate.

As the surface tension makes difference in the metal transfer process but the surface tension coefficient varies with the temperature of the molten metal which is determined by the welding current. So in order to improve accuracy of simulated results and decrease the effect of uncertain surface tension coefficient on the simulated results, the surface tension coefficient should be modified. Thus the relationship between the welding current and the surface tension coefficient is obtained by analysis of regression. The range of the welding current is between $170 \mathrm{~A}$ and $230 \mathrm{~A}$.

$$
\gamma=-0.0216 \exp (I / 43.517)+4.11379
$$

Based on the surface tension coefficient obtained from Eq. (6), the simulated results at $I=210 \mathrm{~A}$ is shown in Fig. 3. The whole metal transfer process which includes the droplet formation, evolution and detachment is similar to Fig. 2. Compared Fig. 3 with Fig. 2, it shows that with the increase of the welding current, the liquid bridge becomes longer and the probability for satellite droplets increases. According 

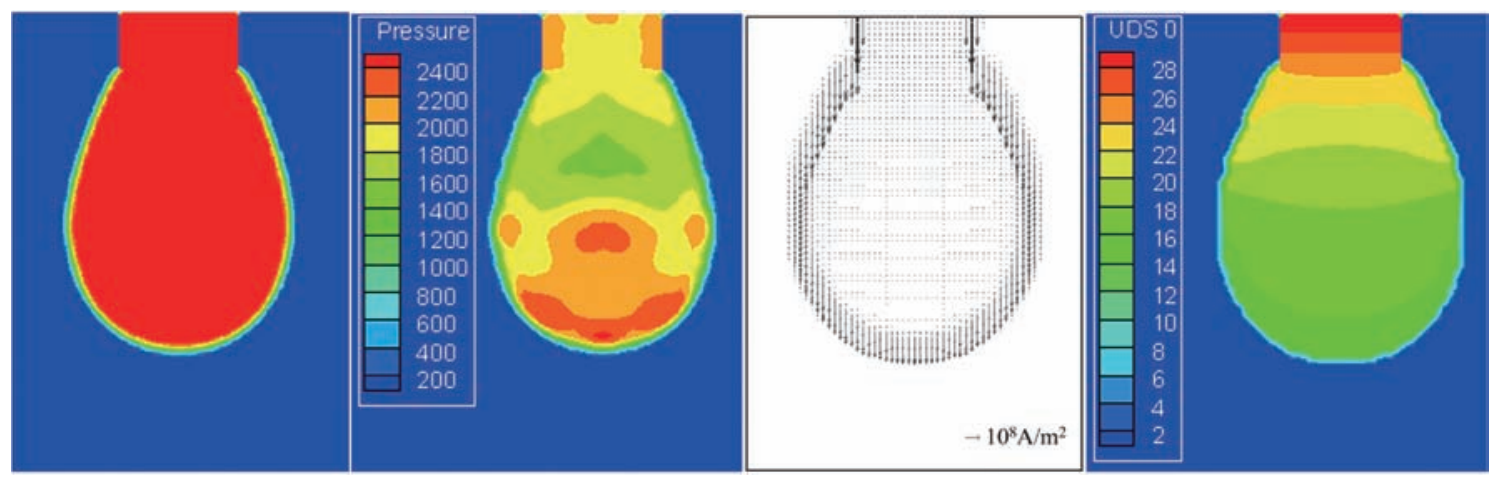

Fig. 4 Description of the physical quantities in the droplet ( $I=175 \mathrm{~A}, t=100 \mathrm{~ms}$ ): (a) droplet profile; (b) pressure; (c) current density; (d) electric potential
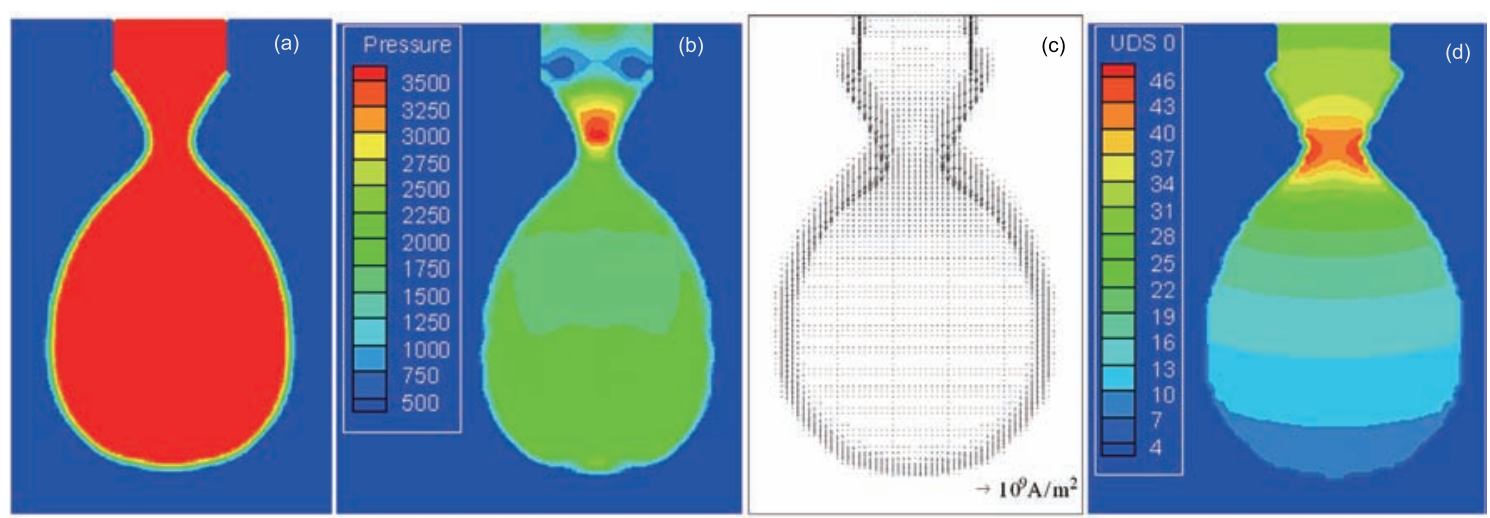

Fig. 5 Description of the physical quantities in the droplet ( $I=175 \mathrm{~A}, t=147.7 \mathrm{~ms}$ ): (a) droplet profile; (b) pressure; (c) current density; (d) electric potential

to Eq. (6), the welding current increases and the surface tension coefficient decreases. The surface tension force pushes upwards but small surface tension is not big enough to hold the molten liquid and accelerates droplet separation. This means that higher welding current elongates the liquid bridge and the probability of the spatter increases ${ }^{[30]}$.

As an example, when $I$ takes 175 A, the descriptions of the physical quantities in the droplet including the pressure, current density and electric potential at two typical stages are shown in Fig. 4 and Fig. 5. Comparing the pressure distribution, we can find that the maximum pressure occurs at the neck of the droplet. Because the curvature at the neck is large, a lot molten metal accumulate there, so it causes that the pressure is very large. The large pressure leads to the molten metal to be extruded through the electrode tip to form a droplet at high velocity and, thus, will accelerate the breakup of the liquid bridge. In the process of droplet growing, the electric potential presents unidimensional distribution and decreases in the axial direction. Because the electrode is in direct current positive, the electrode is at high electric potential and the workpiece is at low electric potential. Then the primary welding current component is in the axial direction so in the axial direc- tion the electric potential decreases. Then when the neck appears, the maximum electric potential is at the neck, where the current density is the highest and the electrical resistance increases largely. So the electric potential at the neck is very high. Due to the high electric potential, a large electromagnetic force is generated that will accelerate the droplet detaching process.

To verify accuracy of the simulated results, welding experiments were performed and a high-speed photography system was used to record the real metal transfer process with a xenon lamp used as a background light source to exclude the high-intensity arc light and then a shadow graph of the droplet could be obtained. The schematic of high-speed photography system is shown in Fig. 6.

The experimental images which stand for one cycle time of the metal transfer process are shown in Fig. 7 and Fig. 8. Three physical quantities including the simulated diameter, length and cycle time of the detached droplet are selected as the criteria to test the accuracy of simulated results. The detailed numeric comparison between simulated results and experimental ones when welding current is at $175 \mathrm{~A}$ and $210 \mathrm{~A}$ are given in Table 3 and Table 4 . In summary, the simulated results for $I=175 \mathrm{~A}$ and $I=210 \mathrm{~A}$ are 
Table 3 Comparisons between the simulated and experimental results $(I=175 \mathrm{~A})$

\begin{tabular}{cccc}
\hline & $\begin{array}{c}\text { Diameter of detached } \\
\text { droplet }(\mathrm{mm})\end{array}$ & $\begin{array}{c}\text { Length of detached } \\
\text { droplet }(\mathrm{mm})\end{array}$ & $\begin{array}{c}\text { Cycle time } \\
(\mathrm{ms})\end{array}$ \\
\hline Simulated result & 2.52 & 3.12 & 148.6 \\
Experimental result & 2.64 & 3.33 & 151 \\
Error & $4.55 \%$ & $6.31 \%$ & $2.24 \%$ \\
\hline
\end{tabular}

Table 4 Comparisons between the simulated and experimental results $(I=210 \mathrm{~A})$

\begin{tabular}{cccc}
\hline & $\begin{array}{c}\text { Diameter of detached } \\
\text { droplet }(\mathrm{mm})\end{array}$ & $\begin{array}{c}\text { Length of detached } \\
\text { droplet }(\mathrm{mm})\end{array}$ & $\begin{array}{c}\text { Cycle time } \\
(\mathrm{ms})\end{array}$ \\
\hline Simulated result & 1.655 & 2.03 & 32 \\
Experimental result & 1.62 & 2.26 & 33 \\
Error & $2.12 \%$ & $10.18 \%$ & $3.03 \%$ \\
\hline
\end{tabular}

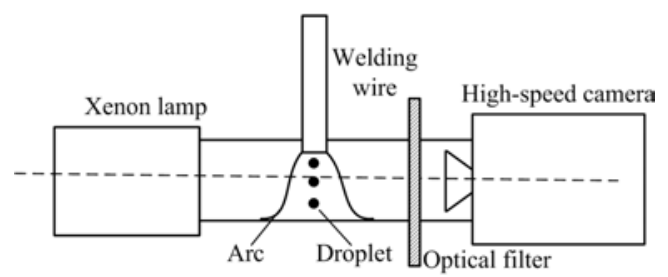

Fig. 6 Schematic of the high-speed photography system
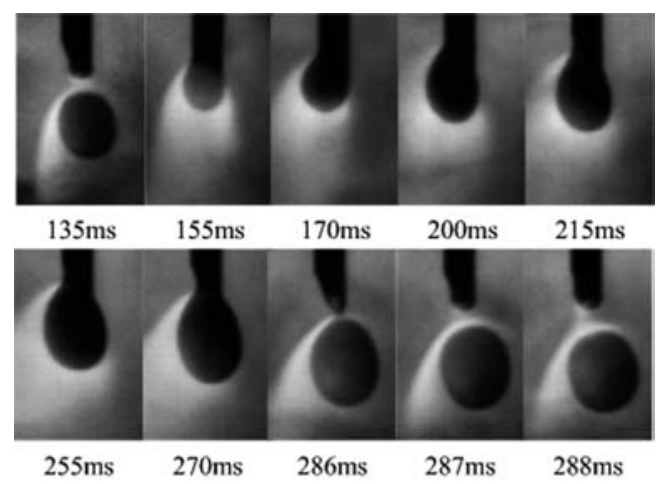

Fig. 7 High-speed photography for $I=175$ A

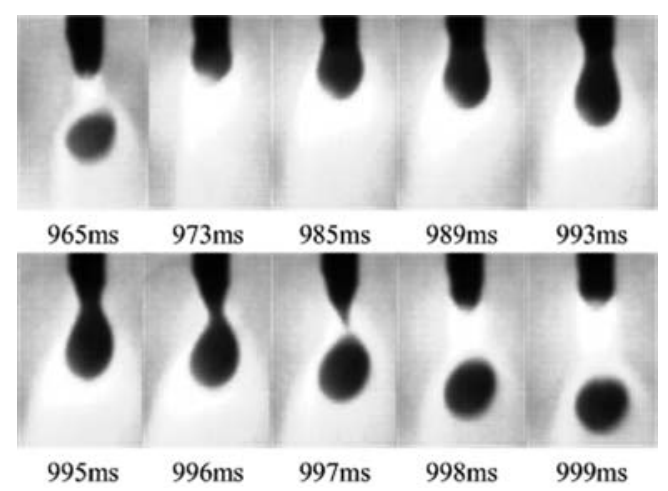

Fig. 8 High-speed photography for $I=210$ A

in reasonable good agreement with the experimental ones. Thus, experimental evidences support the validity of the simulated results.

Fig. 9 shows that there are some discrepancy be-

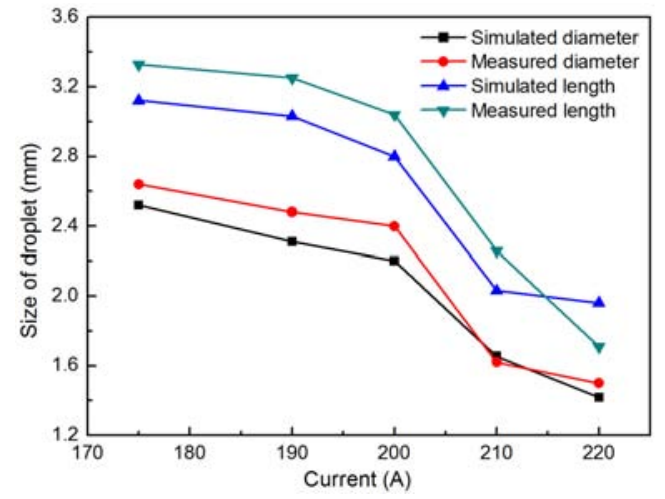

Fig. 9 A comparison between simulated droplet size and experimental measurements

tween the simulated results and the measured ones. The discrepancy perhaps comes from the measurement of the droplet on the high-speed photos. In this model, the inlet velocity is assumed to be the wire feed rate, actually we can not identify accurately the inlet velocity corresponding to the welding current. If the inlet velocity is set larger than the wire feed rate in the real welding process, the volume of the simulated droplet is larger than that of the experimental droplet. So the simulated droplet size will be not accurate. In addition, maybe, we assume the electrode is at the liquid phase without considering the thermal analysis in the simulation and this causes the error. With the increase of the welding current, the discrepancy becomes less. According to Eq. (6), we can find the surface tension coefficient varies with the welding current. In fact, the surface tension plays an important role in the metal transfer process because it is the main attaching force which impedes the metal transfer. Perhaps, at high welding current, the relationship between the surface tension coefficient and the welding current is more applicable, so the simulated results are more accurate.

It is seen in Fig. 10 that there is good agreement between the simulated and measured droplet detachment cycle time. This illustrates the physical model is available and the simulation results are favorable. 


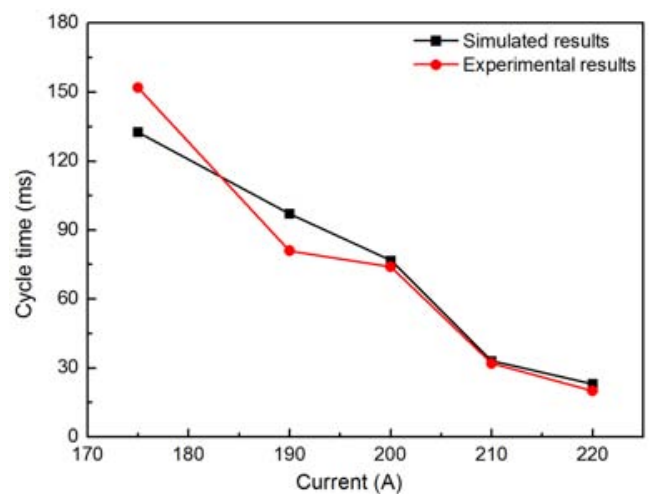

Fig. 10 A comparison between simulated cycle time of the droplet and experimental measurements

\section{Conclusions}

The CFD software FLUENT has been adopted to simulate the metal transfer process in GMAW and the results show that the simulated results are in reasonably good agreement with the experimental ones. In this study, the relationship between the surface tension coefficient and the welding current are modified. In the process of droplet growing, the electric potential presents nearly unidimensional distribution and decreases in the axial direction. At the neck, the pressure, current density and electric potential are the highest. The simulated results agree qualitatively with the experimental ones. In the future, for improving the accuracy of the simulation results, we should combine the arc and thermal analysis with the metal transfer simulation. Meanwhile, we will focus on the metal transfer simulation in twin-electrode welding and laser-arc hybrid welding by adopting FLUENT.

\section{Acknowledgements}

This work was financially supported by the National Natural Science Foundation of China (No. 51175374), the Natural Science Foundation of Tianjin (No. 11JCYBJC06100) and the State Key Lab of Advanced Welding and Joining, Harbin Institute of Technology (No. AWPTM12-08).

\section{REFERENCES}

[1] A. Scotti, V. Ponomarev and W. Lucas, J. Mater. Process. Technol. 212 (2012) 1406.

[2] Y.S. Kim and T.W. Eagar, Weld. J. 72 (1993) 269.
[3] S.K. Choi, J.Y. Lee and C.D. Yoo, Weld. J. 80 (2001) 239.

[4] N. Arif, J.H. Lee, and C.D. Yoo, J. Phys. D 41 (2008) 195503.

[5] N. Arif, J.H. Lee, and C.D. Yoo, J. Phys. D 42 (2009) 035504.

[6] J.H. Choi, J. Lee, and C.D. Yoo, J. Phys. D 34 (2001) 2658.

[7] L.A. Jones, T.W. Eagar and J.H. Lang, J. Phys. D 31 (1998) 107.

[8] C.S. Wu, M.A. Chen and S.K. Li, Comput. Mater. Sci. 31 (2004) 147.

[9] M.A. Chen, C.S. Wu, S.K. Li and Y.M. Zhang, Sci. Technol. Weld. Join. 12 (2007) 10.

[10] U. Ersoy, E. Kannatey-Asibu, S.J. Hu, J. Manuf. Sci. Eng.-Trans. ASME 130 (2008) 061009.

[11] S.W. Simpson, Sci. Technol. Weld. Join. 14 (2009) 262.

[12] Z.H. Rao, S.M. Liao and H.L. Tsai, J. Appl. Phys. 107 (2010) 044902.

[13] B.Y.B. Yudodibroto, M.J.M. Hermans, Y. Hirata, G. den Ouden and I.M. Richardson, Sci. Technol. Weld. Join. 11 (2006) 308.

[14] S.K. Choi, C.D. Yoo and Y.-S. Kim, J. Phys. D 31 (1998) 207.

[15] J. Haidar, J. Phys. D 31 (1998) 1233.

[16] J. Hu and H.L. Tsai, J. Phys. D 100 (2006) 053304.

[17] J. Hu and H.L. Tsai, J. Heat Transf.-Trans. ASME 129 (2007) 1025.

[18] F. Wang, W.K. Hou, S.J. Hu, E. Kannatey-Asibu, W.W. Schultz and P.C. Wang, J. Phys. D 36 (2003) 1143.

[19] H.G. Fan and R. Kovacevic, J. Phys. D 31 (1998) 2929.

[20] M.A. Chen, C.S. Wu and R. Lian, Acta Metall. Sin. 40 (2004) 1227 (in Chinese).

[21] J. Haidar and J.J. Lowke, J. Phys. D 29 (1996) 2951.

[22] T.P. Quinn, M. Szanto, I. Gilad and I. Shai, Sci. Technol. Weld. Join. 10 (2005) 113.

[23] G. Xu, J. Hu and H.L. Tsai, Int. J. Heat. Mass. Trans. 52 (2009) 1709.

[24] G. Wang, P.G. Huang, and Y.M. Zhang, Metall. Mater. Trans. B 34B (2003) 345.

[25] Z.H. Rao, J. Zhou and H.L. Tsai, Int. J. Heat. Mass. Trans. 55 (2012) 6651.

[26] P. Praveen, M.J. Kang, P.K.D.V. Yarlagadda, J. Mater. Process. Technol. 201 (2008) 502.

[27] O. Semenov, V. Demchenko, I. Krivtsun, U. Reisgen, O. Mokrov and A. Zabirov, Model. Simul. Mater. Sci. Eng. 20 (2012) 045003.

[28] J.F. Lancaster, The Physics of Welding, Pergamon Press, Oxford, England, 1983, p.213.

[29] C.W. Hirt and B.D. Nichols, J. Comput. Phys. 39 (1981) 201.

[30] K. Kadota and Y. Hirata, Weld. World. 55 (2011) 50. 